\title{
Immune Transformations in Primary and Secondary Syphilis Patients before and after Treatment and its Clinical Significance
}

\author{
Sedzro Divine Mensah, Li Huiling, Zhang Xiaoli, Wang Yong, Zhang Yanlai, Ma Lei, \\ Gao Rongsheng* \\ Department of Laboratory Medicine, The First Affiliated Hospital of Jiamusi University, Heilongjiang, Province, China
}

\section{ABSTRACT}

Objective: The objective of this study was to determine the levels of interleukin 2 (IL-2), to measure the T cell subpopulations, NK and B cells count in Stage I and II syphilis patients before and after treatment, to analyze the changes in cellular and humoral immunity during the pathogenesis and treatment of the disease, and to further study the role of cellular and humoral immunity during syphilis infection and the relationship between mechanism and prognosis of the disease.

Methods: We divided the experimental subjects into five groups: 100 healthy donors as control, 50 untreated primary syphilis patients, 42 treated primary syphilis patients, 50 untreated secondary syphilis patients, and 46 treated secondary syphilis patients. Fresh anticoagulated blood samples were collected from patients and prepared for T cell subpopulations, B lymphocytes, and NK cell testing using the flow cytometer within $24 \mathrm{~h}$. The serum was separated, and IL-2 levels were measured using the enzyme-linked immunosorbent assay method.

Results: (1) Changes in T-lymphocyte subsets: Comparing the BT I group with the normal, CD4+ T cells increased significantly. In BT II category, the CD4+ T count was significantly low when compared with the control, the CD8+ T cells significantly increased. In AT I group, CD4+ T cells increased significantly. CD4+ T cells reduced in AT II group, CD8+ T cells increased significantly. Comparing AT II and BT II group, the CD4 + T cells of AT II increased significantly, and the CD8 + T cell count of AT II decreased. Comparing BT I and BT II, a significant reduction was observed in CD4+ cells while CD8+ cells increase significantly. CD4+ cells decreased significantly in AT I and AT II comparison, (2) changes in NK cells: There was a significant decrease $(P<0.05)$ in BT I NK cells against the standard. NK cells count significantly $(P<0.05)$ higher in BT II and when compared with the control. Among BT II and AT II, NK cells count decreased significantly $(P<0.05)$ after treatment. There was a significant increase in BT I and BT II NK cells, (3) changes in B cells: B lymphocytes decreased significantly $(P<0.05)$ in BT II. In comparing BT I and BT II, B cells decreased significantly. Similarly, B cells decreased significantly in AT I and AT II groups comparison, (4) changes in IL-2 levels: Significant changes were noted in all groups when compared with the normal. IL-2 decreased significantly when BT I and BT II compared.

Conclusions: T cells, NK cells, and IL-2 are involved in the onset and pathogenic mechanisms of the primary and secondary syphilis disease and these cells influence the treatment of the disease. The efficacy of clinical treatment of primary and secondary syphilis patients may be assessed using IL-2 as an alternative surrogate marker.

Key words: Treponema pallidum (syphilis), T lymphocytes, natural killer cells, interleukin-2, BT I (untreated Type I syphilis patients), AT I (treated Type I syphilis patients), BT II (untreated Type II syphilis patients), AT II (treated Type II syphilis patients)

\section{INTRODUCTION}

Syphilis is a systemic disease caused by a thin, tightly coiled spirochete, Treponema pallidum (TP) subspecies Pallidum. $^{[1]}$ Syphilis is credited to have infected 12 million additional people in 1999, with $>90 \%$ of cases in the developing world. ${ }^{[2]}$ It influences between 700,000 and 1.6 million pregnancies a year, resulting in spontaneous abortions, stillbirths, and congenital syphilis.

${ }^{\star}$ Corresponding author:

Email: sedim65k@yahoo.com

http://dx.doi.org/10.20530/IJIMHS409

ISSN 2056-9866 @ 2017
Transmission of TP can occur through sexual contact, kissing/close connection with active lesions, transfusions, or unintentional injection. The disease is most infectious during primary and secondary syphilis when patients have chancres, mucous patches, condyloma latum, and other lesions which are highly contagious. Primary/Type I/Stage I syphilis is typically acquired by direct sexual contact with the infectious lesions of another person. ${ }^{[3]}$ When primary syphilis is not detected at an earlier stage and treated, the disease gradually progresses to the secondary stage/ Stage II/Type II. Approximately 3-90 days after the initial exposure (average 21 days), a skin lesion, called a chancre, show up at the point of contact. ${ }^{[4]}$ The chancre is classically ( $40 \%$ of the time) a single, firm, painless, and nonitchy skin ulceration with a clear base and sharp boundary between 0.3 and $3.0 \mathrm{~cm}$ in size. ${ }^{[4]}$ The lesion, however, may 
take on almost any form. ${ }^{[3]}$ In the classic form, it evolves from a macule to a papule and finally to an erosion or ulcer. The interaction relation between a huge TP load and the immune response of the host is as a result of secondary syphilis. It is the phase of infection in which there is an extensive dissemination of the pathogen to various parts of the body. It becomes apparent at an average of 6 weeks (range 2-16 weeks) after inoculation. TP infection is most often established when the pathogen enters subcutaneous tissues through microscopic abrasions that occur during sexual intercourse. ${ }^{[5]}$ Increased rates among heterosexuals have occurred in China and Russia since the 1990s. ${ }^{[4]}$ The increase been linked to unsafe sexual practices such as sexual promiscuity, prostitution, and decreasing use of barrier protection. ${ }^{[4,6]}$ When syphilis is not detected early enough and is left untreated; it has a mortality of $8-58 \%$, with a greater death rate in males. The number 1 potent antibiotic developed against syphilis is penicillin. Penicillin remains the first choice for the treatment of syphilis till today. ${ }^{[7]}$ The highest treponemicidal serum concentration of penicillin is $0.36 \mu \mathrm{g} / \mathrm{ml}$ which can eliminate the organism in 6-9 h. ${ }^{[8]}$ Human infections with TP elicit dynamic immune reactions causing damage to the body. Several researchers have shown that T cells, B lymphocytes, NK cells, and IL-2 may participate in the pathogenesis of the spirochete. However, their specific response in primary and secondary syphilis remains unclear. ${ }^{[9]}$

\section{Interleukin $2^{[10]}$}

IL-2 is a Th1 cytokine that plays a main role in the triggering and build-up of lymphocytes that have been primed by antigens. The current study shows that TP clearance depends on the Th1 cell-mediated immune response but during early and secondary syphilis infection, there is Th1/Th2 imbalance, and immune cells are inhibited. ${ }^{[1]}$ As a result of this immune suppression, the body's ability to clear the TP reduces and this also affects the outcome of the treatment and the disease development. According to some scholars, cytokines IL- 2 and IFN- $\gamma$ can inhibit the expression of Th2 cytokine IL-10. ${ }^{[11]}$ Th1, therefore, plays a major role in the response of lymphocytes to the clearance of the syphilis antigen. IL-2 signals through the IL-2 receptor, sophistication made up of three chains, termed alpha, beta, and gamma. IL-2 also advances the differentiation of $\mathrm{T}$ cells into effector $\mathrm{T}$ cells and memory $\mathrm{T}$ cells when the initial $\mathrm{T}$ cell is stimulated by a foreign body (antigen), thus helping the body to clear pathogens. ${ }^{[12]}$ Its expression and secretion are tightly regulated and functions as part of both transient positive and negative feedback mechanism in mounting immune responses and tamping them down. ${ }^{[13]}$ Even though IL-2 is a Th1 cytokine, it is a crucial regulator of Th2 in a STAT5-dependent manner and an inhibitor Th17 differentiation/development. IL-2 is a major serum biomarker in several pathological situations such as cancer, infectious diseases, transplant contradiction, varied sclerosis, rheumatoid arthritis, systemic lupus erythematosus, and Type I diabetes.

\section{METHOD}

\section{Type of study design and venue}

This research was conducted at the Dermatology and Clinical Laboratory Department of the First Affiliated Hospital of Jiamusi University, Heilongjiang Province, China. The whole study lasted for 12 months. Approval was sought from the Ethics Committee of The First Affiliated Hospital of Jiamusi University, China. All participants gave their written consents. The patients were identified and referred for enrollment by the dermatologists after careful examination and evaluation. Diagnosis of syphilis at the various stages of infection was done by an amalgamation of compatible history, clinical markers, and the results of non-treponemal and treponemal tests of peripheral blood. 100 healthy donors and hospital staff, who visited the outpatient department of dermatology for a medical check-up for STD prevention, were recruited for this work. All control subjects were nonreactive for HIV and blood tests for the spirochete in both serum RPR and TP antibody test, (Baxter Healthcare Corp., Miami, USA), and have no clinical symptoms consistent with TP disease. Syphilis patients were not included in the research if they had received an antibiotic within 1 month before their first visit in the case of new diagnosis as well as any of the following conditions: HIV-positive, pregnant women, and children, recent infections including colds, diabetes, fever, pericoronitis, and autoimmune diseases, allergic diseases such as asthma, allergic rhinitis, measles, tumor patients, and other associated conditions that may interfere with our study result.

\section{Specimen collection and follow-up protocol}

Syphilis patients chosen for the study visited the dermatology department from March 2015 to February 2016 and satisfied the criteria for the research. Blood samples were taken into the EDTA tubes after which centrifugation and serum preservation in freezer at $-80^{\circ} \mathrm{C}$ for later use was done. Patients with the history of syphilis or syphilis treatment were carefully monitored and accurate data on previous treatment RPR titer values assessed. Medical history and preserved blood samples of patients who were already treated and were visiting the hospital for check-up were available at the doctor's table and the immunology laboratory department, respectively. Serum TP antibody and RPR test in both new cases and follow-up cases were used for the diagnosis. All syphilis patients had their blood taken every 3 months for RPR tests. The grouping for our research was as follows: Group 1: Normal control group, the hospital healthy population, serum RPR (-), and serological TPPA/TP (Chemclin 600, Beijing Chemclin Biotech Co., Ltd, China) antibody test (-), Group 2: Untreated primary 
syphilis patients (BT I), presence of skin lesion called chancre at the point of contact such as the penis, cervix, and genitals 3-6 weeks without treatment, serum PRP (+), and serological TPPA/TP antibody test (+), Group 3: Untreated secondary syphilis patients (BT II), the presence of a symmetrical, reddish-pink, non-itchy rash on the trunk and extremities; evident 6 weeks after inoculation and commonly disseminated including the palms and soles, serum PRP (+), and serological TPPA/TP antibody test (+), Group 4: Confirmed and treated primary syphilis patients (AT I), serum PRP (+), and serological TP antibody test (+), and Group 5: Confirmed and treated secondary syphilis patients (AT II), serum PRP (+), and serological TP antibody test (+).

\section{Flow cytometry and enzyme-linked immunosorbent assay (ELISA) assay}

With flow cytometry (BD FACSCanto IIV33896002182, 2014, USA) using CD monoclonal antibodies labeled by BD Multitest 6-color TBNK reagent (Becton, Dickinson Company, United States), and unique feature of peripheral blood lymphocyte immunophenotypes was detected through pre-treatment and post-treatment of 188 cases of syphilitic patients and 100 cases of normal persons. The test was conducted within $24 \mathrm{~h}$. Meanwhile, the same patients' serum IL-2 level was measured by Human IL-2 ELISA Kit (E-10146, Shanghai Guangrui Biological Technology Co. Ltd, China) and Perlong Microplate reader (DNM-9606, Nanjing Perlove Medical Equipment Co., Ltd, China) was used to read the absorbance at $450 \mathrm{~nm}$ within $15 \mathrm{~min}$.

\section{Statistical analysis}

The Chi-square test and Student $t$-test were used. Figures were presented as mean plus or minus the standard error of the mean. All statistical analyses were performed using SPSS 21.0 software. The significance level was set at $P<0.05$.

\section{RESULTS}

Comparing the immune functions of primary syphilis patients before treatment with control persons, a significant increase $(P<0.05)$ was detected in $\mathrm{CD} 4+$ cells. There was an increase in $\mathrm{CD} 8+, \mathrm{CD} 4+/ \mathrm{CD} 8+$ ratio, and B lymphocytes in BT I, but the increase was not statistically significant $(P>0.05)$. Nevertheless, there was a significant decrease $(P<0.05)$ in NK cells and IL-2 produced in BT I when compared with the control. There was a significant difference in the ages of the control subjects and the BT I patients. CD3+ count in BT I patients decreased $(P>0.05)$. Among secondary syphilis patients, comparing BT II with the normal control, there was a significant $(P<0.05)$ decrease in $\mathrm{CD} 4+, \mathrm{CD} 4+/ \mathrm{CD} 8+$, and B lymphocytes. However, significant increases $(P<0.05)$ were observed in IL-2, CD8+, and NK cells. A decrease was noted in CD3+ count but not significant $(P>0.05)$.
Comparing the control persons with treated primary syphilis patients, there were increases in $\mathrm{CD} 3+, \mathrm{CD} 4+$, CD8+, and B lymphocytes but not statistically significant $(P>0.05)$. A significant increase $(P<0.05)$ was observed only in CD4+ cells. There was a decrease in NK cells and CD4+/CD8+ ratio but not significant. The IL-2 measured after treatment was $105.3 \pm 13.7 \mathrm{pg} / \mathrm{ml}$ and statistically significant when compared with the standard which is 40.9 $\pm 2.4 \mathrm{pg} / \mathrm{ml}$. Again, the comparison between treated Type II syphilis patients and the control population revealed a significant increase $(P<0.05)$ and decrease in $\mathrm{CD} 8+$, and $\mathrm{CD} 4+/ \mathrm{CD} 8+$ ratio, respectively. CD4+, B cells, and NK cells all reduced in count but the reduction is statistically not significant $(P>0.05)$. On the other hand, $\mathrm{CD} 3+$ cells in total increased but not statistically significant $(P>0.05)$. After treatment, the level of IL-2 was still high $(75.3 \pm 12.5 \mathrm{pg} / \mathrm{ml})$, the increase was statistically significant $(P<0.05)$ when compared with the control.

Comparing the immune cells and IL-2 before and after treatment in Type I syphilis patients, there was no significant difference $(P>0.05)$ among the $\mathrm{CD} 3+, \mathrm{CD} 4+, \mathrm{CD} 8+, \mathrm{CD} 4+/$ $\mathrm{CD} 8+, \mathrm{B}$ cells, and NK cells generated before and after treatment, but there was a significant decrease $(P<0.05)$ in the IL-2 produced before and after treatment $(269.3 \pm$ $36.5 \mathrm{pg} / \mathrm{ml}$ and $105.3 \pm 13.7 \mathrm{pg} / \mathrm{ml}$, respectively). Significant increases $(P<0.05)$ were observed in $\mathrm{CD} 3+, \mathrm{CD} 4+$, and CD4+/CD8+ ratio of BT II and AT II after treatment. $B$ cells increased after treatment but not significant. More so, there was a decrease in NK cells after the treatment, and the decrease was statistically significant $(P<0.05)$. After treatment IL-2 and CD8+ cells decreased, but the decrease was statistically not significant $(P>0.05)$. In general, age and gender have no significant effect on the tested parameters.

Comparing BT I and BT II, a significant decrease was observed in CD4+ cells while a significant increase was detected in CD8+ cells. NK cells increased significantly after analyzing BT I and BT II groups. B cells decreased significantly when BT I and BT II were analyzed. IL-2 concentration reduced significantly $(P<0.05)$ when the disease progress from Stage I-II without treatment. CD4+ cells decreased significantly but CD8+ cells increased $(P>0.05)$ in AT I and AT II groups. B cells decreased significantly AT I and AT II groups were studied. NK cells increased in AT I and AT II comparison, but the difference was not statistically significant. IL-2 reduced when Type I and II patients were compared after treatment, but the reduction was not statistically significant.

In general, there was no significant difference observed in the gender of both patients and normal control persons, but there was a strong relationship with age and lymphocyte count. The lymphocyte count decreases with increasing age (Table 1). 


\section{DISCUSSION}

TP, the disease-causing agent responsible for syphilis remains one of the human pathogens that cannot be grown in vitro till present although there are lots of researchers going on regarding that. ${ }^{[14]} \mathrm{TP}$ invasion causes several immunological effects and plays important roles in the incidence of syphilis, development, and recovery. Currently, syphilis immune mechanism is not fully understood, studies have shown that cellular immunity mediated by $\mathrm{T}$ lymphocytes of the TP is crucial, and also cytokines which play an important role. ${ }^{[15]}$ According to experiments conducted by Fitzgerald et al. ${ }^{[2]}$ in a rabbit model to explain the phenomenon of syphilis progression, TP stimulates the body to produce a cellular immune response that is Th1-like responses to get rid of a large number of spirochetes. The activated Th1 cells produce high levels of IL-2 and macrophages to produce large amounts of prostaglandins which have a direct inhibitory effect on the Th1 activity. Th1 response deals with the cellular immunity while the Th2 response concerns the humoral response. When the disease is not diagnosed at an earlier stage and treated, the inhibitory effects of the Th1 cytokines suppress the cellular immunity of the body making the spirochete persist and progress to the secondary stage. The present study was to determine the specific roles played by the lymphocyte subpopulations and IL-2 during infection, prognosis, treatment, and its clinical significance. The study also looks at the effects of IL-2 on both cellular and immune response during syphilis infection.

The general decrease in CD3+ cells (Table 2) of both primary and secondary syphilis patients suggested that the infection had reduced or weakened the cellular immunity of the patients, and this supports earlier works that the total number of $\mathrm{T}$ lymphocytes was reduced in patients with early syphilis. ${ }^{[16]}$ Turner suggested that the T lymphopenia may be as a result of uneven distribution of lymphocytes in the lymphoid system. ${ }^{[17]}$ It has also been suggested that cytotoxic antibodies to T lymphocytes may occur, although lymphocytotoxic antibodies were shown to be active only at $15^{\circ} \mathrm{C}$. There is also the possibility that the TP itself is directly toxic to $\mathrm{T}$ lymphocytes. ${ }^{[18]}$ Nevertheless, there were no significant increases in CD8+, CD4/CD8+ cells, and $\mathrm{B}$ lymphocytes during primary syphilis. The major increase in CD4+ cells during the early stages of the disease stimulates the production of Th1 cytokines such as IL-2. As a result, the level of IL-2 (Table 2) in the peripheral rises significantly $(P<0.05)$. Th1 cells (CD4+ cells) secreted cytokines (IL-2) mainly mediate cellular immune responses. Th2 cytokines (IL-4, IL-5) mainly play a role in humoral immune response in producing antibodies to mediate eosinophil and Th1 antagonism. ${ }^{[19]}$ The balance of Th1/Th2 plays a key role in clearing syphilis. ${ }^{[20]}$

In BT II, the significant $(P<0.05)$ increase in CD8+ cells may be due to the long duration of the disease condition and its suppressive effects on the cellular immunity. Because the cellular immunity was unable to eliminate the pathogen, the spirochetes multiply significantly as a result of a decreased level of IL-2 leading type II syphilis. Since IL-2 is known to be the most important $\mathrm{T}$ cell growth factor which mainly increases the number and activity of $\mathrm{T}$ lymphocytes leading to clearance of the TP pathogen, ${ }^{[21]}$ a decrease in IL-2 may, therefore, account for the significantly low CD4+ cells count and ultimately the CD4+/CD8+ ratio in BT II. Although in our research, the quantity of IL-2 produced in BT II is significantly higher $(P<0.05)$ than the control, it is significantly lower $(P<0.05)$ compared to that produced in BT I syphilis.

IL-2 mainly causes T-cell proliferation, stimulate B cell growth, and antibody production. ${ }^{[11]} \mathrm{NK}$ cells proliferation to clear the blood of TP was significantly inhibited $(P<0.05)$ as the spirochetes multiply spontaneously and the ability of the cells to produce IL-2 decreased. According to Vivier, NK cells provide rapid responses acting at around 3 days after infection triggering cytokine release, causing lysis or apoptosis. ${ }^{[22]}$ Tabor et al. ${ }^{[23]}$ reported that treponemal infection induced the production of suppressor $\mathrm{T}$ cells $(\mathrm{CD} 8+)$, which inhibited macrophage activity. Although both CD4+ cells and CD8+ cells increased

Table 1: Basic information, RPR, and TP test results on the patients and the control

\begin{tabular}{|c|c|c|c|c|c|}
\hline Parameters & BT I & BT II & AT I & AT II & Control \\
\hline $\mathrm{n}$ & 50 & 50 & 42 & 46 & 100 \\
\hline \multirow[t]{2}{*}{ Gender } & $\mathrm{M}(20) \mathrm{F}(30)$ & $\mathrm{M}(28) \mathrm{F}(22)$ & $M(18) F(24)$ & $M(26) F(20)$ & $M(52) F(48)$ \\
\hline & $\mathrm{M}(40 \%) \mathrm{F}(60 \%)$ & $\mathrm{M}(56 \%) \mathrm{F}(44 \%)$ & $\mathrm{M}(43 \%) \mathrm{F}(58 \%)$ & $\mathrm{M}(56.5 \%) \mathrm{F}(43.5 \%)$ & $\mathrm{M}(52 \%) \mathrm{F}(48 \%)$ \\
\hline \multirow[t]{2}{*}{ Mean age (years) } & $44.2 \pm 2.394$ & $39 \pm 3.463$ & $40.4 \pm 3.396$ & $34.696 \pm 2.494$ & $35.9 \pm 0.893$ \\
\hline & $M(51.7) F(39.1)$ & M (39.3) F (38.5) & M (39.4) F (38.8) & $M(36.4) F(31.8)$ & $M(35.6) F(36.2)$ \\
\hline \multirow[t]{3}{*}{ RPR } & $28(56 \%) \Phi$ & $34(68 \%) \Phi$ & $4(9.5 \%) \Phi$ & $6(13 \%) \Phi$ & Nil \\
\hline & $22(44 \%)^{*}$ & $16(32 \%)^{*}$ & $38(90.5 \%)^{*}$ & $36(78.3 \%)^{*}$ & \\
\hline & & & & $4(8.7 \%) \#$ & \\
\hline \multirow[t]{2}{*}{ TP antibody } & 50 (positive) & 50 (positive) & 42 (positive) & 42 (positive) & 100 (negative) \\
\hline & & & & 4 (negative) & \\
\hline
\end{tabular}

$\Phi=$ Patients whose RPR test results are $\geq 1: 8,{ }^{*}$ Patients whose RPR test results are $<1: 8$ (not infectious), \#patients whose RPR test results are non-reactive 
Table 2: Average values of T cells, B cells, NK cells, and IL-2 of syphilis patients and the normal expressed as (mean \pm standard error of the mean) and comparisons

\begin{tabular}{|c|c|c|c|c|c|c|c|c|}
\hline Group & Age & IL-2 & CD3+ & CD4+ & CD8+ & CD4+/CD8+ & B cells & NK cells \\
\hline Control & $36.5 \pm 1.31$ & $40.9 \pm 2.4$ & $62.67 \pm 1.49$ & $37.84 \pm 0.99$ & $19.51 \pm 1.05$ & $2.13 \pm 0.18$ & $13.54 \pm 0.776$ & $14.90 \pm 1.27$ \\
\hline BT I & $44.2 \pm 2.40$ & $269.3 \pm 36.5$ & $61.05 \pm 2.30$ & $41.87 \pm 1.52$ & $21.45 \pm 1.32$ & $2.34 \pm 0.28$ & $15.45 \pm 1.46$ & $2.34 \pm 0.28$ \\
\hline$P$ value & $0.006^{*}$ & $0.000 *$ & 0.601 & $0.029 *$ & 0.287 & 0.573 & 0.220 & $0.000 *$ \\
\hline AT I & $38.71 \pm 2.96$ & $105.3 \pm 13.7$ & $64.71 \pm 1.03$ & $42.42 \pm 0.30$ & $24.19 \pm 2.40$ & $2.00 \pm 0.12$ & $13.996 \pm 0.75$ & $11.67 \pm 1.18$ \\
\hline$P$ value & 0.490 & $0.000 *$ & 0.334 & $0.000 *$ & 0.087 & 0.587 & 0.681 & 0.059 \\
\hline BT I and AT I ( $P$ value) & 0.211 & $0.002^{\circ}$ & 0.119 & 0.710 & 0.333 & 0.302 & 0.413 & 0.808 \\
\hline BT II & $38.96 \pm 3.46$ & $99.5 \pm 21.3$ & $59.91 \pm 1.71$ & $26.58 \pm 0.46$ & $31.72 \pm 1.31$ & $0.87 \pm 0.04$ & $10.29 \pm 0.598$ & $19.00 \pm 1.202$ \\
\hline$P$ value & 0.564 & $0.018^{*}$ & 0.258 & $0.000 *$ & $0.000 *$ & $0.000 *$ & $0.001^{*}$ & $0.012^{*}$ \\
\hline AT II & $35.44 \pm 2.52$ & $75.3 \pm 12.5$ & $65.99 \pm 1.55$ & $35.35 \pm 0.90$ & $28.96 \pm 1.19$ & $1.26 \pm 0.052$ & $11.38 \pm 0.833$ & $12.64 \pm 0.988$ \\
\hline$P$ value & 0.737 & $0.017^{*}$ & 1.640 & 0.080 & $0.000 *$ & $0.000 *$ & 0.720 & 0.190 \\
\hline BT II and AT II ( $P$ value) & 0.308 & 0.281 & $0.012^{\circ}$ & $0.000^{\circ}$ & 0.990 & $0.000^{\circ}$ & 0.319 & $0.010^{\circ}$ \\
\hline BT I and BT II ( $P$ value) & 0.222 & $0.000^{\circ}$ & 0.714 & 0.000 & 0.000 & 0.000 & 0.003 & 0.000 \\
\hline AT I and AT II ( $P$ value) & 0.372 & 0.221 & 0.535 & 0.000 & 0.063 & 0.000 & 0.011 & 0.554 \\
\hline
\end{tabular}

BT I: Type I/Stage I/Phase I/primary syphilis patients without treatment, AT I: Type I/Stage 1/Phase I/primary syphilis patients after treatment, BT II: Type II/Stage II/phase II/secondary syphilis patients without treatment, AT II: Type II/Stage II/Phase II/secondary syphilis patients after treatment, * $<0.05$ (statistically significant differences when group compared with control), $<0.05$ (statistically significant differences when group comparison was done)

during infection, the significant increase observed in CD4+ cells as compared to CD8+ cells explains why the increase was detected in CD4/CD8+ cell ratio. Early TP infection induces both humoral and cellular immunity of the body. ${ }^{[24]}$ Despite the significant increase in CD4+ cells before treatment, the body was not able to clear itself of the spirochete. The humoral immunity confers protection on the body whiles the cellular immunity is weakened. The significant difference in the ages of the normal and the BT I group could be as a result of the higher diversity of the control group which was randomly chosen.

There is a close relationship between cytokines related to Th1 and Th2 imbalance, and in our present study, after TP infection progresses to the secondary stage; there is a paradigm shift from Th1 to Th2, which may be important for clinical diagnosis and treatment of the disease. Li's research suggested that Th2 cytokines such as IL-4 dominates in Stage II syphilis and induces the growth and differentiation of B cells for antibody production. Contrary to our result in BT II, there was a significant reduction $(P<0.05)$ in B lymphocytes. NK cells, on the other hand, increased significantly and this may enhance their killing function (Figure 1). It is, therefore, evident from our study that NK cells play a major role in "fighting" the spirochete when the cellular and humoral immunity was suppressed. Lower levels of IL-2 promote the growth and differentiation of NK cells and are therefore the major cells fighting the pathogen in cases of suppressed CD4+ activity and/or low IL-2 production. NK cells are known to cross-talk with dendritic cells to impact $\mathrm{T}$ cell responses. NK cells shape the subsequent immune response by either boosting or dampening macrophage and/or T cell responses through IFN-gamma or IL-10. This activity of NK cells might be the cause of the suppressed cell immunity whenever the NK is high.

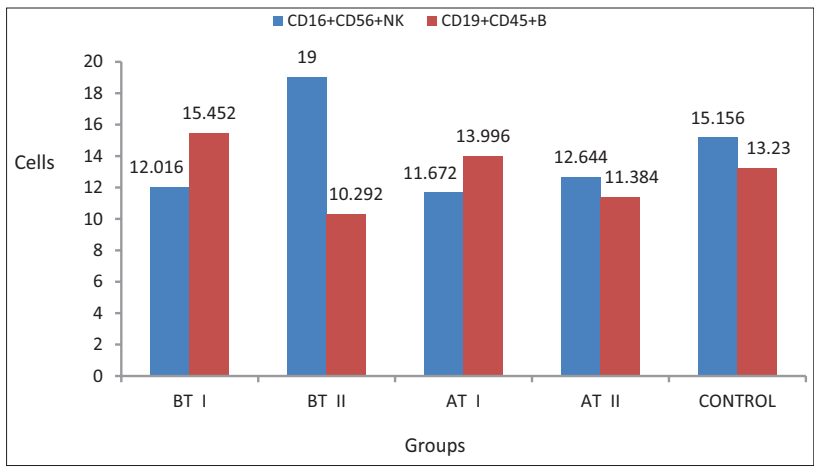

Figure 1: NK and B cells in AT I, AT II, BT I, BT II, and control.

After treatment, there was an increase in CD3+ cells and a significant $(P<0.05)$ increase in $\mathrm{CD} 4+$ cells in primary syphilis, and this simply means that the treatment was effective. There was no significant increase in B lymphocytes, and an insignificant decrease was observed in NK cells (Figure 1). This reiterates the fact that cellular immunity played the major role in clearing the pathogen even after treatment. Among the type II patient after treatment (AT II), the increase in $\mathrm{CD} 4+$ cells counts indicated that the treatment was effective. There was a decrease in CD4+, B lymphocytes, NK cells, and CD4+/ $\mathrm{CD} 8+$ cell ratio when compared with the control; this could mean that these cells are "currently" involved in the active clearing of the spirochete hence the decrease in their numbers. The quantity of IL-2 in Type II syphilis after treatment was still high $(P=0.170)$ and is likely to reduce and stabilize with time.

When BT I and AT I groups were compared, there was no significant difference in all the cellular parameters tested (Figure 2), this may be as a result of the short period of type I syphilis, early diagnosis and treatment given. As in 
the case of many infections, the body's own natural immune response can eliminate TP independent of antibiotic usage, ${ }^{[14]}$ but in states of immune compromise, even the most effective antibiotics may fail to eradicate TP. ${ }^{[25]} \mathrm{In}$ Stage I syphilis, the body's cellular immunity has been compromised by TP, and the body cannot completely clear the TP. After treatment, CD4+ cells significantly increase the secretion of cytokines, an example of which is IL-2. The IL-2 is most important T-cell growth factor mainly produced by Th1 cells which can increase the number and activity of $\mathrm{T}$ lymphocytes leading to clearance of the TP pathogen. The higher the level of IL-2 in the patient's body, the ability of the body to get rid of the TP is stronger. ${ }^{[26]}$

When the comparison was done between BT II and AT II, a significant increase $(P<0.05)$ was noted in CD3+ cells, CD4+ cells, and CD4+/CD8+ cell ratio (Figure 2). An increase was detected in B lymphocytes but not statistically significant $(P=0.319)$. The population of these cells increases as a result of the penicillin treatment and these cells finally aid in clearing the spirochete from the body. The action of the suppressor cells (CD8+) was impeded with active proliferation and activity of helper $\mathrm{T}$ cells (CD4+). IL-2 values decrease after a successful treatment, but the decrease was not significant when compared with untreated Type II syphilis patients. NK cells reduced significantly $(P<0.05)$ after treatment and may be due to its quick and short period response. The activity of NK cells may become less important after enough of the spirochetes have been cleared from the body. According to Vivier et al., NK cells provide rapid responses acting at around 3 days after infection triggering cytokine release, causing lysis or apoptosis. ${ }^{[22]}$ Yang Nitto ${ }^{[6]}$ postulated that cellular immune suppression and reduction is one of the main factors in patients with prolonged and unhealed syphilis. He suggested that in addition to the regular antisyphilitic treatment should be combined with certain immune modulators to enhance cellular immunity, efficacy, and reduce recurrence.

In AT I, 90.5\% of the patients (Table 1) recorded a significant decrease in their RPR test although they all tested positive for TP antibody after treatment. According to Romanowski, most patients who have reactive treponemal tests will have reactive tests for the remainder of their lives irrespective of treatment or disease activity. On the other hand, ${ }^{[14]}$ Fantry suggests that non-treponemal tests in patients with primary syphilis should be non-reactive within 6-12 months after treatment. Relating these two hypotheses to our result, it could be that these patients will forever test positive to the TP antibody or may test negative for the antibody with time although the treatment is a success. From Figure 3, it can be deduced that total lymphocyte count decreases with increasing age of the patients, and this could mean that longer treatment duration with immune boosters is needed in the elderly syphilis patients for a quick healing.

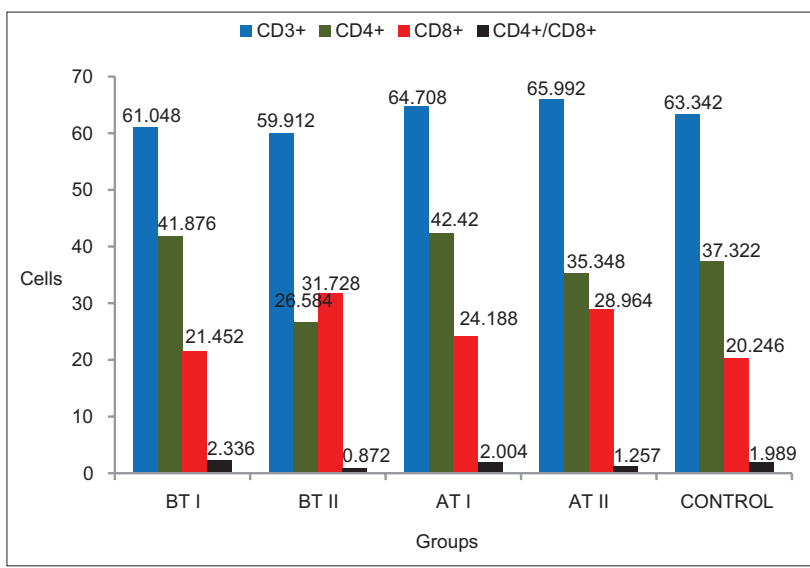

Figure 2: $\mathrm{CD} 3+, \mathrm{CD} 8+, \mathrm{CD} 4+$, and $\mathrm{CD} 4+/ \mathrm{CD} 8$ in AT I, AT II, BT I, BT II, and control.

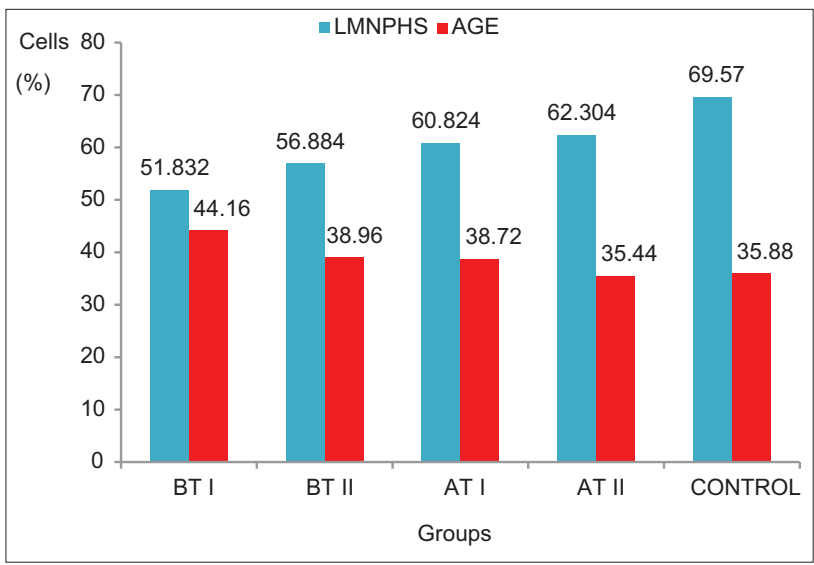

Figure 3: Age and LMNPHS in BT I, BT II, AT I, AT II, and control.

From our study, it can be implied that when syphilis is not diagnosed at an earlier stage, Th1-type immune response gradually weakens, Th2-type immune response dominates eventually leading to Th1/Th2 immune imbalance. This phenomenon results in an increased level of Th2 cytokines such as IL-4 and decreases in Th1 cytokines, and this suppresses the body's cellular immunity and ability to clear TP, thereby causing the disease to persist. Cellular immune suppression and reduction are one of the main factors in patients with prolonged and unhealed syphilis. We suggested that in addition to the regular antisyphilitic treatment, certain immune modulators should be combined to enhance cellular immunity, efficacy, and reduce recurrence. ${ }^{[5]}$

In conclusion, our study has shown that cellular immunity becomes dominant within very short period but unable to clear the body of the pathogen. The body produces a high amount of IL-2 on spirochete infection for the proliferation and activation of the cellular immunity. Treatment has a closer relationship with CD4+ cells and IL-2 and without treatment; the disease will ultimately progress to the $2^{\text {nd }}$ stage. The function and activities of CD4+ cells and IL-2 are directly proportional while IL-2 and B cells are inversely related (Figure 4). Humoral immunity mediated by B cells 


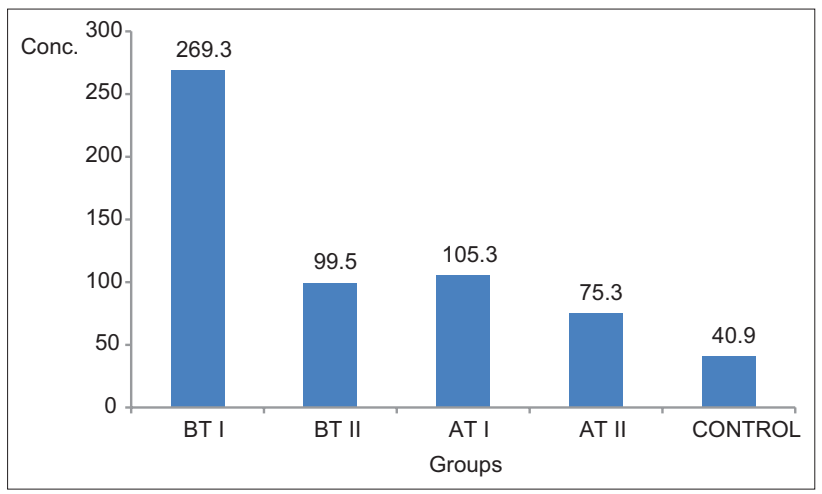

Figure 4: Concentrations of interleukin 2 in peripheral blood of BT I, BT II, AT I, AT II patients, and control.

plays no significant role in type I syphilis before and after treatment. IL-2 may be used as an alternative surrogate marker for assessing the efficacy of clinical treatment of primary syphilis patients. NK cells were the only major cells aiding the body to clear the pathogen in BT II. Our findings support the hypothesis that human infection with TP is followed by a complex interaction between cellular and humoral immunity, the former being suppressed in the primary stage but reactivated after successful treatment.

\section{ACKNOWLEDGMENTS}

We thank the Management and Dermatology and Laboratory Department of the First Affiliated Hospital of Jiamusi University, China, for their support.

\section{REFERENCES}

1. Tramont EC. Treponema pallidum (syphilis). Principles and Practice of Infectious Diseases. $7^{\text {th }}$ ed. Philadelphia, PA: Elsevier Churchill Livingstone; 2009.

2. Stamm LV. Global challenge of antibiotic-resistant Treponema pallidum. Antimicrob Agents Chemother 2010;54:583-9.

3. Pickering LK, Baker CJ, Kimberlin DW, Long SS, editors. Red Book: 2006 Report of the Committee on Infectious Diseases. Elk Grove Village Illinois (IL): American Academy of Pediatrics; 2006.

4. Kent ME, Romanelli F. Reexamining syphilis: An update on epidemiology, clinical manifestations, and management. Ann Pharm 2008;42:226-36.

5. LaFond RE, Lukehart SA. Biological basis for syphilis. Clin Microbiol Rev 2006;19:29-49.

6. Ficarra G, Carlos R. Syphilis: The renaissance of an old disease with oral implications. Head Neck Pathol 2009;3:195-206.

7. Fitzgerald TJ. Effects of cefetamet (Ro 15-8074) on Treponema pallidum and experimental syphilis. Antimicrob Agents Chemother 1992;36:598-602.

8. Eagle H, Fleischman R, Musselman AD. The effective concentrations of penicillin in vitro and in vivo for streptococci, pneumococci, and Treponema pallidum. J Bacteriol 1950;59:625.

9. Wang C, Zhu L, Gao Z, Guan Z, Lu H, Shi M, et al. Increased interleukin-17 in peripheral blood and cerebrospinal fluid of neurosyphilis patients. PLoS Negl Trop Dis 2014;8:e3004.

10. Arenas-Ramirez N, Woytschak J, Boyman O. Interleukin-2: Biology, design and application. Trends Immunol 2015;36:763-77.

11. Boyman O, Sprent J. The role of interleukin-2 during homeostasis and activation of the immune system. Nat Rev Immunol 2012;12:180-90.

12. Liao W, Lin JX, Leonard WJ. IL-2 family cytokines: New insights into the complex roles of IL-2 as a broad regulator of $\mathrm{T}$ helper cell differentiation. Curr Opin Immunol 2011;23:598-604.

13. Malek TR, Castro I. Interleukin-2 receptor signaling: At the interface between tolerance and immunity. Immunity 2010;33:153-65.

14. Fantry L, Tramont EC. Treponema pallidum (syphilis). Antimicrobial Therapy and Vaccines. $2^{\text {nd }}$ ed., Vol. 200. New York, NY: Apple Trees Productions; 2002. p. 749-757.

15. Fitzgerald $\mathrm{T}$. The Th1/Th2-like switch in syphilitic infection: Is it detrimental? Infect Immunity 1992;60:3475-9.

16. Jensen JR, From E. Alterations in $\mathrm{T}$ lymphocytes and T-lymphocyte subpopulations in patients with syphilis. Br J Vener Dis 1982;58:18-22.

17. Turner D, Wright D. Lymphadenopathy in early syphilis. J Pathol 1973;110:305-8.

18. de Jong N, Koehorst JA, van der Sluis J, Boer AM. Autolymphocytotoxins in syphilis. $\mathrm{Br} \mathrm{J}$ Vener Dis 1980;56:297-301.

19. Luckheeram RV, Zhou R, Verma AD, Xia B. CD4区T cells: Differentiation and functions. Clin Dev Immunol 2012;2012:925135.

20. Song JQ, Liu SW. Immunological Changes in Syphilitic Paitents with Different Courses.

21. Lin CC, Gao X, Chen XS, Chen Q, Cohen MS. China's syphilis epidemic: A systematic review of seroprevalence studies. Sex Transm Dis 2006;33:726-36.

22. Vivier E, Raulet DH, Moretta A, Caligiuri MA, Zitvogel L, Lanier LL, et al. Innate or adaptive immunity? The example of natural killer cells. Science 2011;331:44-9.

23. Tabor DR, Kiel DP, Jacobs RF. Cyclophosphamide-sensitive activity of suppressor $\mathrm{T}$ cells during treponemal infection. Immunology 1987;62:127-32.

24. Arroll TW, Centurion-Lara A, Lukehart SA, Van Voorhis WC. T-cell responses to treponema pallidum subsp. Pallidum antigens during the course of experimental syphilis infection. Infect Immun 1999;67:4757-63.

25. Hook EW $3^{\text {rd }}$, Baker-Zander SA, Moskovitz BL, Lukehart SA, Handsfield $\mathrm{HH}$. Ceftriaxone therapy for asymptomatic neurosyphilis. Case report and western blot analysis of serum and cerebrospinal fluid igG response to therapy. Sex Transm Dis 1986;13:185-8.

26. Liebowitz DN, Lee KP, June CH. Costimulatory approaches to adoptive immunotherapy. Curr Opin Oncol 1998;10:533-41. 